\title{
Virus replicon particle based Chikungunya virus neutralization assay using Gaussia luciferase as readout
}

Sabine Gläsker ${ }^{1}$, Aleksei Lulla ${ }^{2}$, Valeria Lulla ${ }^{2}$, Therese Couderc ${ }^{3,4}$, Jan Felix Drexler ${ }^{1}$, Peter Liljeström ${ }^{5}$, Marc Lecuit ${ }^{3,4,6}$, Christian Drosten ${ }^{1}$, Andres Merits ${ }^{2}$ and Beate Mareike Kümmerer ${ }^{1 *}$

\begin{abstract}
Background: Chikungunya virus (CHIKV) has been responsible for large epidemic outbreaks causing fever, headache, rash and severe arthralgia. So far, no specific treatment or vaccine is available. As nucleic acid amplification can only be used during the viremic phase of the disease, serological tests like neutralization assays are necessary for CHIKV diagnosis and for determination of the immune status of a patient. Furthermore, neutralization assays represent a useful tool to validate the efficacy of potential vaccines. As CHIKV is a BSL3 agent, neutralization assays with infectious virus need to be performed under BSL3 conditions. Our aim was to develop a neutralization assay based on non-infectious virus replicon particles (VRPs).

Methods: VRPs were produced by cotransfecting baby hamster kidney-21 cells with a CHIKV replicon expressing Gaussia luciferase (Gluc) and two helper RNAs expressing the CHIKV capsid protein or the remaining structural proteins, respectively. The resulting single round infectious particles were used in CHIKV neutralization assays using secreted Gluc as readout.

Results: Upon cotransfection of a CHIKV replicon expressing Gluc and the helper RNAs VRPs could be produced efficiently under optimized conditions at $32^{\circ} \mathrm{C}$. Infection with VRPs could be measured via Gluc secreted into the supernatant. The successful use of VRPs in CHIKV neutralization assays was demonstrated using a CHIKV neutralizing monoclonal antibody or sera from CHIKV infected patients. Comparison of VRP based neutralization assays in 24- versus 96-well format using different amounts of VRPs revealed that in the 96-well format a high multiplicity of infection is favored, while in the 24-well format reliable results are also obtained using lower infection rates. Comparison of different readout times revealed that evaluation of the neutralization assay is already possible at the same day of infection.
\end{abstract}

Conclusions: A VRP based CHIKV neutralization assay using Gluc as readout represents a fast and useful method to determine CHIKV neutralizing antibodies without the need of using infectious CHIKV.

Keywords: Chikungunya virus, Virus replicon particles, Neutralization assay, Gaussia luciferase

\footnotetext{
* Correspondence: kuemmerer@virology-bonn.de

${ }^{1}$ Institute of Virology, University of Bonn Medical Centre, Bonn, Germany

Full list of author information is available at the end of the article
} 


\section{Background}

Chikungunya virus (CHIKV) is an arthropod-borne, enveloped virus of the genus Alphavirus, family Togaviridae [1]. The single-stranded RNA genome of positive polarity is capped at the 5' end and polyadenylated at the 3 ' end. It is 12 kilobases $(\mathrm{kb})$ in length and contains two open reading frames. The 5 ' two-thirds of the genome are directly translated into the nonstructural proteins (nsP1-4) whereas the structural proteins (capsid, E1 and E2) and two small cleavage products (E3 and 6k) are translated from a subgenomic RNA, which corresponds to the last third of the genome [1]. Due to the fact that alphaviruses exhibit a broad host range (including avian, insect and mammalian cells), high levels of protein expression and a small genome that is easy to manipulate, they have been exploited as vectors for foreign gene expression or vaccination [2]. This is especially the case for Sindbis virus (SINV), Semliki Forest virus (SFV), and Venezuelan equine encephalitis virus (VEEV) [3-5]. In this context, production of virus replicon particles (VRPs) has been described, which allows the expression of foreign genes via so called single round infectious particles. Besides VRP systems, which are composed of an alphavirus replicon and one helper RNA that encodes all structural genes, VRP systems have been established in which the capsid and envelope helper regions are encoded by two separate helper RNAs $[4,6,7]$. This reduces the likelihood of recombination and renders the production of replicationproficient viruses (RPVs) negligible, thereby increasing biosafety $[6,7]$.

CHIKV was first described in 1953 during an outbreak in Tanzania, East Africa $[8,9]$. It came into focus again due to large-scale epidemics in the Indian Ocean region starting 2005/2006 [10,11]. Since then further recurring epidemics have been observed from time to time in Africa, Indian Ocean Islands, and many parts of South-East Asia and virus emergence also occurred in European countries, like Italy and France [12-16]. CHIKV is transmitted to humans by culicids of the genus Aedes including Aedes aegypti and Aedes albopictus [17-19]. Infection with the virus results in Chikungunya fever (CHIKF), which is characterized by high fever, fatigue, headache, nausea, vomiting, rash, myalgia and severe arthralgia $[8,20,21]$. To date, there is no specific treatment and no licensed vaccine available against infection with CHIKV.

To determine the immune status of a patient, a neutralization (NT) assay is necessary. Furthermore, NT assays represent an important method to validate the efficiency of potential vaccines during vaccine development. Most neutralization assays described so far for CHIKV involve the use of infectious CHIKV particles. Besides plaque reduction neutralization assay, NT assays using immunofluorescence or inhibition of the cytopathogenic effect (CPE) as readouts have been described [22-26]. Recently, a CHIKV pseudotyped lentiviral vector-based NT assay was established [27]. The latter circumvents the use of infectious CHIKV particles and readout was performed several days after transduction by measuring luciferase activity in the cells transduced with the CHIKVpseudotyped lentiviral vector [27]. The aim of our studies was to also develop an NT assay for CHIKV infection, which is independent of infectious particles but in addition allows a very fast and easy readout. Several reporter proteins have been described, which allow to follow up viral infection or replication. Besides fluorescent proteins reporter proteins based on bioluminescent reactions are widely used. The latter include for example Renilla (R) or Firefly (F) luciferase (luc) but also Gaussia luciferase (Gluc), which in contrast to Rluc and Fluc is secreted into the supernatant thereby facilitating analyses [28,29]. Hence, we aimed to establish a CHIKV VRP based NT assay using the oxidative decarboxylation of coelenterazine catalyzed by secreted Gluc leading to emission of blue light as readout.

\section{Results}

Establishment of CHIKV replicon and helper constructs

For the production of CHIKV replicon particles a split helper system should be applied since it has been described that providing the structural proteins via two separate helper constructs did not result in detectable incidental production of infectious particles due to recombination $[6,7]$.

A CHIKV replicon was established, in which the structural genes were replaced by a reporter gene (Figure 1A). For easy readout, Gluc was chosen as reporter protein, since it is secreted into the supernatant and therefore allows easy readout without the need of preparing cell lysates. Furthermore, two helper plasmids were established expressing either the CHIKV capsid protein $\mathrm{C}$ (pChikHelper-C) or the CHIKV envelope proteins p626K-E1 (pChikHelper-E). The helper RNAs contain the 5' and 3' CHIKV replication signals and a subgenomic promoter followed by either the capsid gene or the remaining structural proteins, respectively (Figure 1A). The helper RNAs as well as the CHIKV replicon RNA can be produced in vitro from plasmids, which contain these sequences under control of an SP6 promoter.

To prove efficient secretion of Gluc from the replicon in vitro transcribed replicon RNA was electroporated into baby hamster kidney-21 (BHK-21) cells. After electroporation, increasing amounts of Gluc could be detected over time in the supernatant demonstrating the efficient replication and secretion of Gluc of the established CHIKV replicon (Figure 1B).

\section{Conditions for optimized VRP production}

For production of VRPs the CHIKV Gluc replicon was coelectroporated with the two helper RNAs into BHK- 


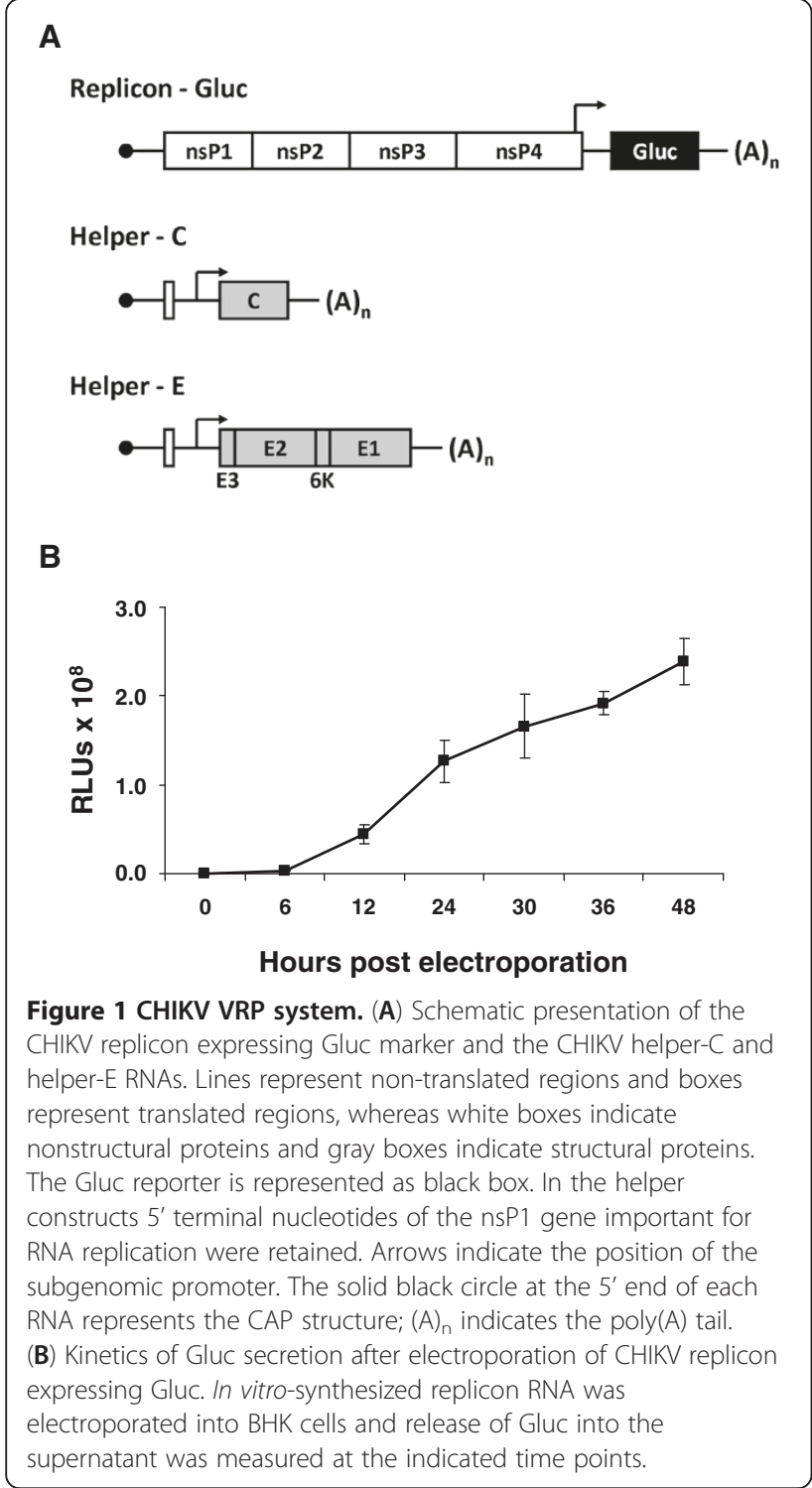

21 cells. It has been observed that VRP production is more efficient at temperatures lower than $37^{\circ} \mathrm{C}$. Hence, to compare VRP production at different temperatures, coelectroporated cells were incubated at either $37^{\circ} \mathrm{C}$ or $32^{\circ} \mathrm{C}$ and the activity of secreted Gluc was measured in Relative Light Units (RLUs). Whereas at $37^{\circ} \mathrm{C}$ Gluc secretion increased over time until $48 \mathrm{~h}$ post electroporation before dropping down again, Gluc levels remained lower and more constant at lower temperature (Figure 2A). Interestingly, when the supernatants were passaged once on BHK cells to analyze for produced VRPs it turned out that VRPs had been released to higher and more constant levels for the experiment performed at $32^{\circ} \mathrm{C}$ (Figure 2B). This suggests that although Gluc was more efficiently secreted at $37^{\circ} \mathrm{C}$ the VRP production was indeed more stable at lower temperature. Hence, for optimized VRP production, the

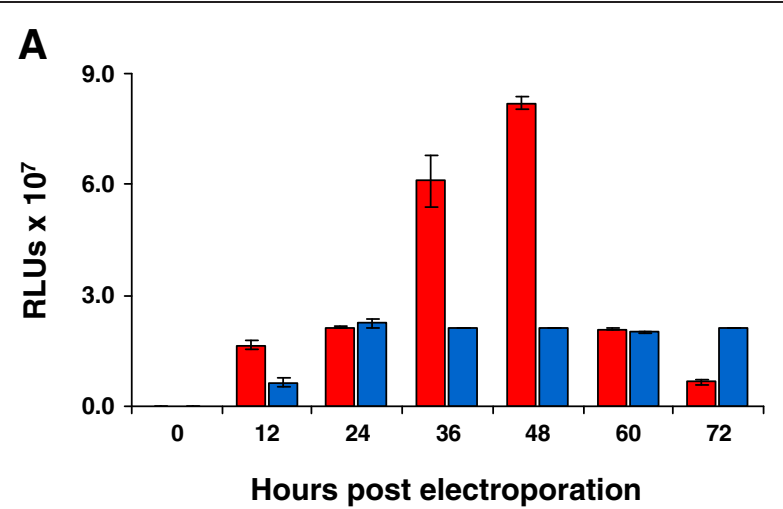

B

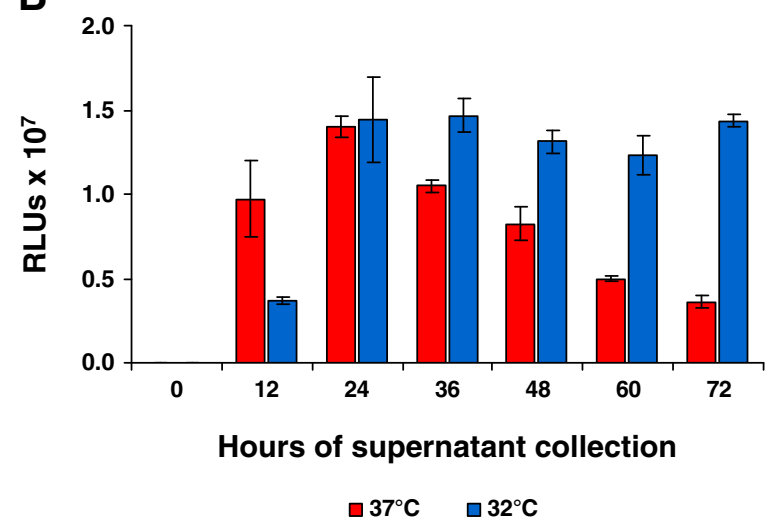

Figure 2 Optimum of VRP production. (A) In vitro-synthesized Gluc replicon RNA was coelectroporated with in vitro-synthesized helper-C and helper-E RNA and cells were either incubated at $32^{\circ} \mathrm{C}$ or $37^{\circ} \mathrm{C}$. Supernatants were harvested at the indicated time points after electroporation and Gluc activity was measured in RLUs. (B) Same volumes of supernatants harvested at different time points from the different incubation temperatures in $(\mathbf{A})$ were used to infect fresh $\mathrm{BHK}$ cells at $37^{\circ} \mathrm{C}$. At $6 \mathrm{~h}$ post infection, Gluc activity was measured in the supernatant.

coelectroporated cells were incubated at $32^{\circ} \mathrm{C}$ and VRPs were harvested at $36 \mathrm{~h}$ post electroporation. Harvested VRPs were purified via a sucrose cushion. As determined by CHIKV real-time PCR, the 30 -fold concentrated VRP stocks contained around $1 \times 10^{9}$ viral RNA copies $/ \mathrm{ml}$.

\section{Comparison of test conditions with regard to VRP} infection rate, plate format and readout time

For comparable analyses same test conditions need to be applied. These include among others parameters like the amount of VRPs used in the NT assay as well as the readout time or the plate format used. Even though the VRP concentration in established stocks can be determined via real-time PCR to calculate defined infection rates it might be desirable to use the same VRP batch for as many NT assays as possible. NT assays using different amounts of VRPs corresponding to multiplicities of infection (MOIs) of 5, 0.5 and 0.05 calculated based on the determined 
RNA copies in the VRP stock were evaluated. Another reason for testing also lower MOIs was that this better reflects the conditions used in classical plaque neutralization assays. There, a low MOI needs to be chosen to be able to count single plaques for readout. Evaluation of different MOIs was first carried out using the neutralizing monoclonal antibody (mAb) D3.62 directed against CHIKV E2 (T. Couderc and M. Lecuit, unpublished data). The respective experiments were performed in either a 24- or 96-well format and readout was done at 6 and $24 \mathrm{~h}$ post VRP infection (Figure 3). To evaluate the neutralization activity, the infectivity rates measured via Gluc secretion retained after VRP incubation with the respective antibody dilution were determined in percent compared to the VRP only control.
As shown in Figure 3, stepwise neutralization was observed after applying the monoclonal antibody in different dilutions. In the 24-well format the most consistent data were obtained when VRPs were used at MOI 5 or MOI 0.5 . Decreasing the MOI to 0.05 increased the deviation from the mean. For the 96-well plate format MOI 5 yielded the most reliable data whereas the number of outliers increased at lower MOI. The least reliable data were obtained in the 96-well format using an MOI of 0.05. Comparing the data received after evaluating the NT assay at $6 \mathrm{~h}$ post infection (p.i.) versus $24 \mathrm{~h}$ p.i. revealed similar results indicating that the readout of the Gluc VRP assay readily can be performed within one day.

Similar results were obtained when the same set of conditions was tested using a human serum from a CHIKV

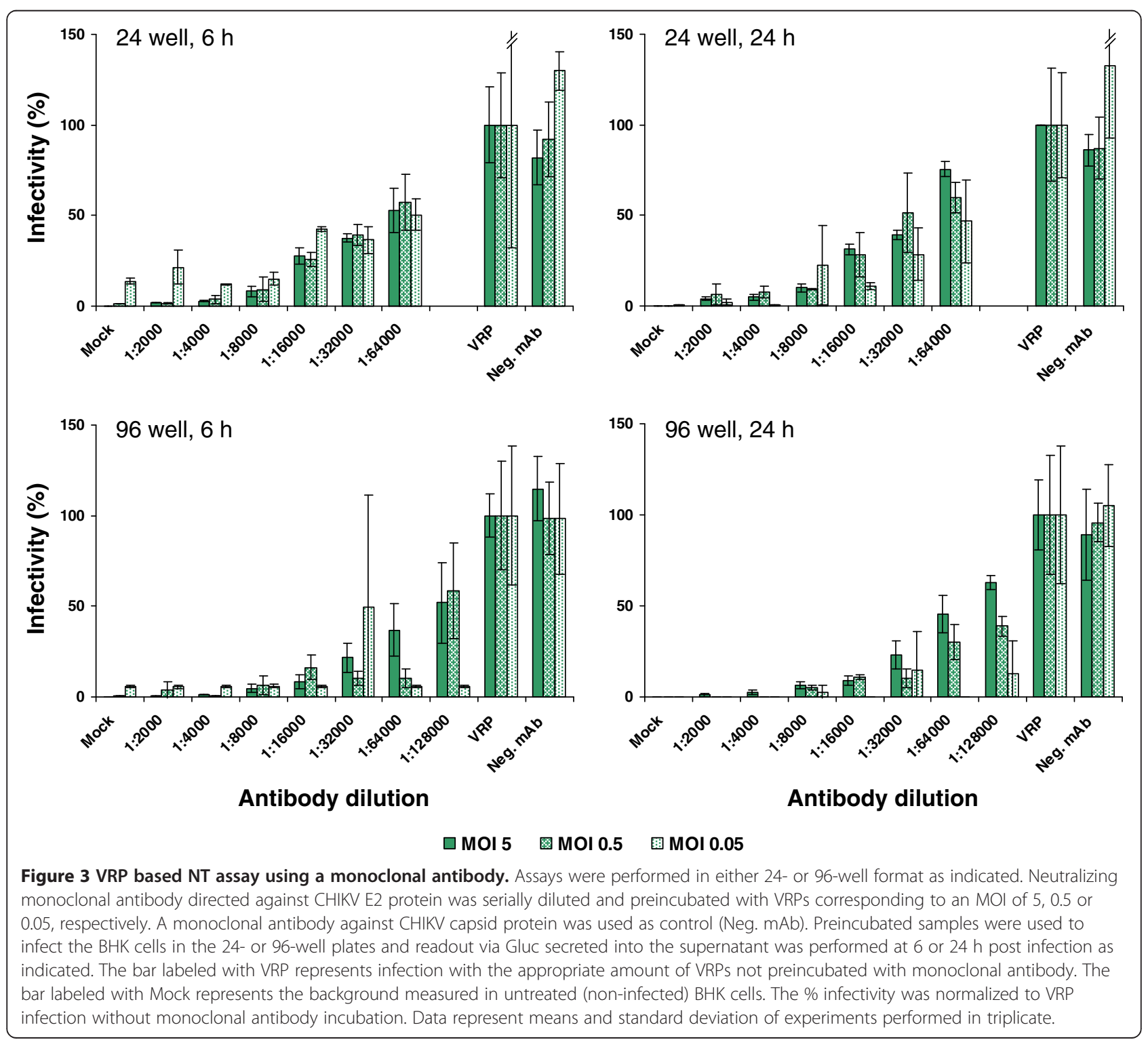


patient (1753/06) (Figure 4). Using immunofluorescence analysis, the serum was confirmed to be positive against CHIKV (see also Figure 5A).

Whereas the human CHIKV patient serum resulted in dose dependent neutralization, a negative serum from a healthy person did not show any neutralization activity (Figure 4). Again, MOI 5 and MOI 0.5 turned out to be the most applicable conditions in the 24-well format and MOI 5 yielded the most consistent results in the 96-well format (Figure 4).

\section{Evaluation of VRP based neutralization assay in} comparison to plaque neutralization assay

To further evaluate the applicability of the established VRP based NT assay comparison to a classical plaque neutralization assay based on infectious CHIKV was drawn. The respective analysis was done using again the neutralizing monoclonal antibody D3.62, the patient serum 1753/06, as well as two further sera (662/06 and 575/06) from CHIKV patients diseased during the 2006 epidemic in the Indian Ocean region [30]. All sera as well as the monoclonal antibody were confirmed to be reactive against CHIKV using indirect immunofluorescence (Figure 5A). The plaque neutralization assay was performed in a 6-well plate format using 100 plaque forming units (PFU) per well (Figure 5B), whereas for the VRP based NT assay both the 24-well and 96-well format was applied using MOI 5 (Figure 5C). The neutralization activity of a serum is usually judged by determining the serum dilution, which results in 50 or $80 \%$

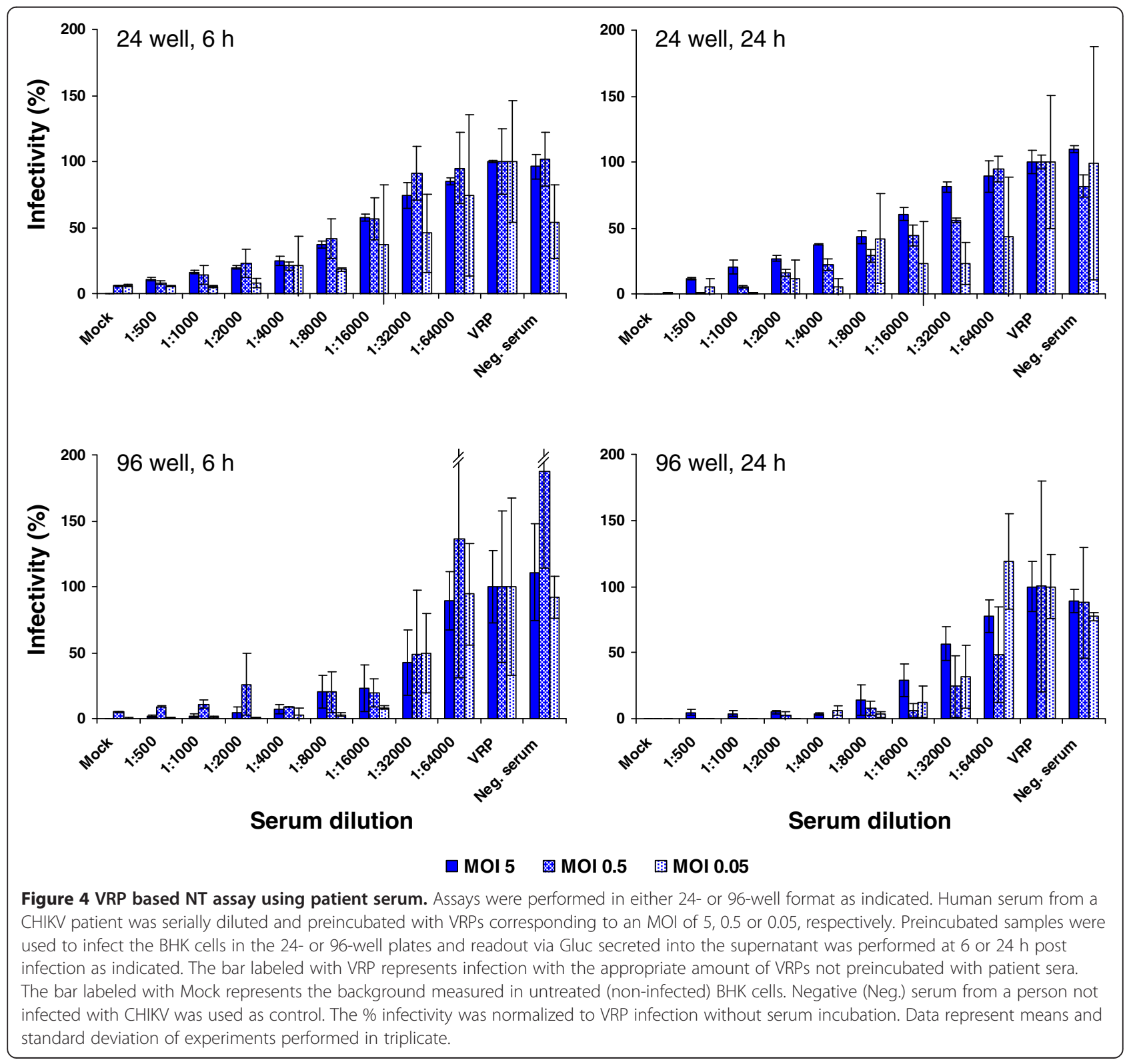




\section{A}
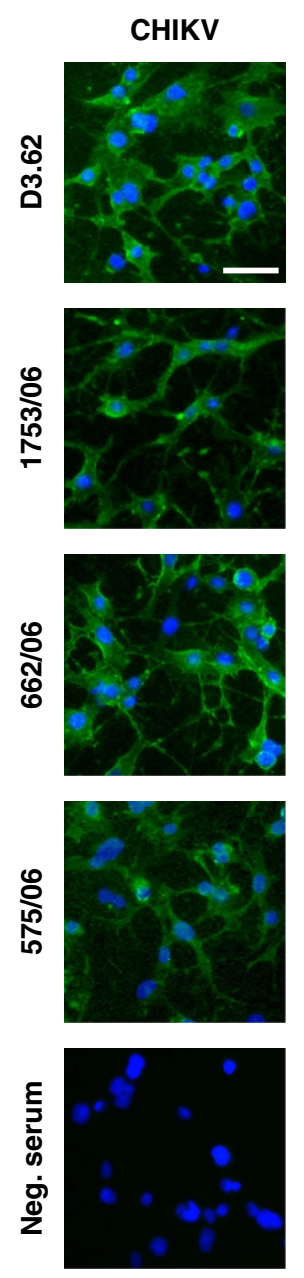
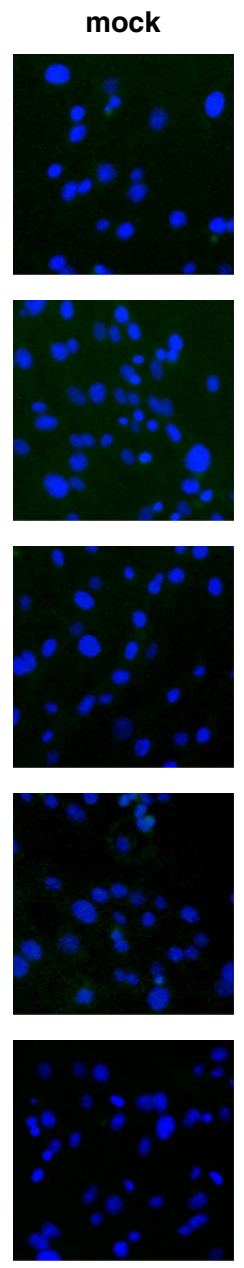

B

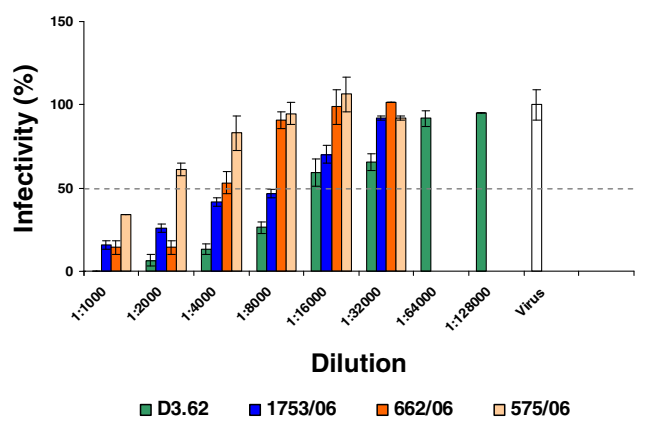

C
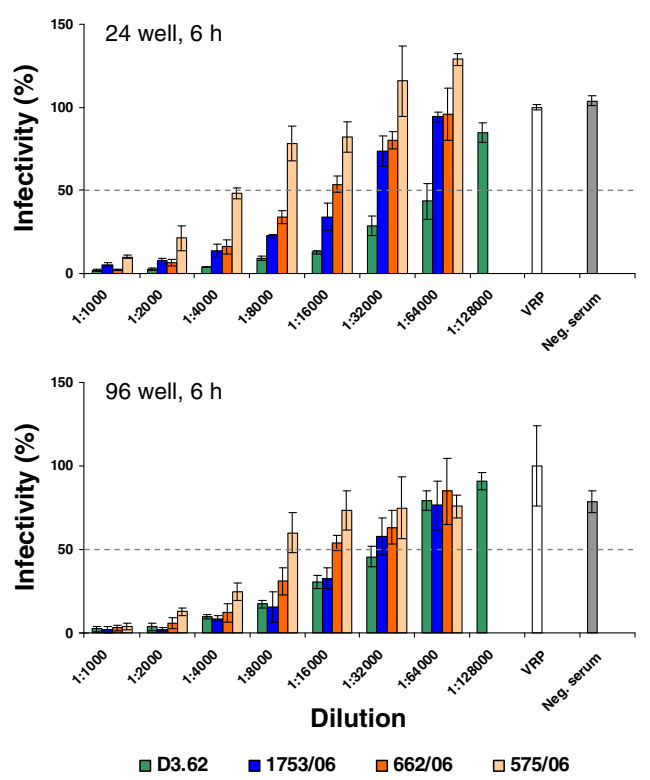

Figure 5 Comparison of plaque neutralization and VRP based NT assays. (A) CHIKV infected and non-infected (mock) BHK cells were used for indirect immunofluorescence analyses using the indicated monoclonal antibody or human sera. Nuclei were stained with DAPI. The bar represents $25 \mu \mathrm{m}$. (B) For the plaque neutralization assay, serial dilutions of monoclonal antibody D3.62 or three patient sera were preincubated with infectious CHIKV. NT assays were performed in 6-well format with readout at $48 \mathrm{~h}$ p.i. using crystal violet staining. The bar labeled with Virus represents a non-neutralized infectivity control, which was set $100 \%$. The $\%$ infectivity was normalized to the non-neutralized virus infection. The gray dotted line indicates the 50\% infectivity threshold. Data represent the means and ranges of duplicate infection experiments. (C) For the VRP based NT assay, serial dilutions of monoclonal antibody D3.62 or three patient sera were preincubated with VRPs. NT assays were performed in either 24-well plates (top panel) or 96-well plates (bottom panel) using VRPs at an MOI of 5. Readout was performed at $6 \mathrm{~h}$ p.i. via measurement of Gluc secreted into the supernatant. The bar labeled with VRP represents infection with the appropriate amount of VRPs not preincubated with patient sera/antibody. Negative (Neg.) serum from a person not infected with CHIKV was used as control. The \% infectivity was normalized to VRP infection without serum/antibody incubation. The gray dotted line indicates the $50 \%$ infectivity threshold. Data represent average and standard deviation of experiments performed in triplicate.

reduction of infectivity $\left(\mathrm{NT}_{50}\right.$ or $\mathrm{NT}_{80}$ titer, respectively). When using different methods of NT assays, comparison of absolute $\mathrm{NT}_{50}$ values is limited due to different test conditions. However, the order of neutralizing activity among antisera tested should be the same. Table 1 provides details on the predicted $\mathrm{NT}_{50}$ values using the data sets depicted in Figure 5B and 5C. Overall, the $\mathrm{NT}_{50}$ titers determined via the classical plaque neutralization assay were lower than those observed in the VRP assays (Table 1). Nevertheless, the same order with regard to neutralizing activity of the samples was obtained for all NT assay types, with the monoclonal antibody neutralizing the best and serum 575/06 neutralizing the least (Table 1). This comparison was supported by narrow 95\% confidence intervals (CI) determined for each serum (Table 1). In the 96-well VRP assay format, all 95\% CI overlapped partially. In contrast, the 95\% CI overlapped marginally only for two sera in the plaque assay, and no overlap at all was observed in the 24-well VRP assay format. Hence, the CHIKV VRP based NT 
Table 1 Neutralizing antibody titers $\left(\mathrm{NT}_{50}\right)$

\begin{tabular}{|c|c|c|c|c|c|c|}
\hline \multirow[b]{2}{*}{$\mathrm{mAb} /$ Serum } & \multicolumn{2}{|c|}{ Virus-NT ${ }^{a}$} & \multicolumn{2}{|c|}{ VRP-NT $\left(24\right.$ well) ${ }^{b}$} & \multicolumn{2}{|c|}{ VRP-NT (96 well) ${ }^{b}$} \\
\hline & $\mathrm{NT}_{50}^{c}$ & $(95 \% \mathrm{CI})^{e}$ & $\mathrm{NT}_{50}^{d}$ & $(95 \% \mathrm{Cl})$ & $\mathrm{NT}_{50}^{d}$ & $(95 \% \mathrm{Cl})$ \\
\hline D3.62 & 16256 & $(13465-19731)$ & 58306 & $(45196-79754)$ & 26885 & $(22799-32054)$ \\
\hline $1753 / 06$ & 5978 & $(4882-7350)$ & 16203 & $(12911-20881)$ & 26314 & $(21004-34401)$ \\
\hline $662 / 06$ & 3582 & $(2581-5061)$ & 13437 & $(11819-15382)$ & 18743 & $(15050-24194)$ \\
\hline $575 / 06$ & 1375 & $(615-2183)$ & 4511 & $(3590-5662)$ & 10911 & (7743-15876) \\
\hline
\end{tabular}

a Neutralization assay performed with virus particles (plaque assay).

$b$ Neutralization assay performed with virus replicon particles (Gluc assay).

$c \mathrm{NT}_{50}$ values were determined via probit analysis using the data set of Figure 5B.

$d \mathrm{NT}_{50}$ values were determined via probit analysis using the data sets of Figure $5 \mathrm{C}$.

e $95 \%$ confidence interval.

assay represents a useful alternative to CHIKV NT assays involving infectious virus and in addition allows a fast and easy analysis of neutralizing antibodies in serum samples that can be performed within one day.

\section{Discussion}

The present study shows that Gluc expressing VRPs represent a useful tool to determine neutralizing antibodies without the need of infectious CHIKV particles. VRPs were produced by cotransfection of a CHIKV replicon expressing Gluc and two helper RNAs. First studies describing the production of VRPs involved the cotransfection of a single helper RNA encoding all structural proteins $[3,31]$. However, in this constellation a single RNA recombination event is sufficient to reproduce a full-length genome resulting in production of infectious particles [31]. Expressing the structural proteins via two helper RNAs circumvents this problem and it was shown that using this strategy production of infectious particles due to recombination is negligible $[2,6]$, which is an important aspect with regard to biosafety issues.

For certain alphaviruses, like SFV and SINV, expression of the structural proteins was described to be dependent on an enhancer sequence located in the 5' terminal part of the sequence encoding the capsid protein $[32,33]$. To ensure in these cases efficient translation of the envelope proteins in a bipartite helper packaging system, the enhancing sequence of the capsid protein was fused 5'-terminally to the envelope genes [6,7]. However, for VEEV a split helper system was described in which the envelope proteins were expressed without a capsid translation enhancer [4]. The latter implicates that the enhancer sequence is not necessarily needed in the VEEV context [4]. This seems to be also the case for $\mathrm{CHIKV}$, as no stable hairpin structure comparable with capsid enhancers of SFV and SINV could be predicted in the corresponding region of the CHIKV genome. Nevertheless, it was also observed that the CHIKV replicon could be efficiently packed using split helper RNAs of SFV (A. Merits and A. Lulla, unpublished data). Therefore the effect of classical SFV capsid enhancer was tested also in the context of CHIKV helper RNAs. In this experiment the presence of enhancer sequence not only failed to increase but reduced the VRP production (A. Lulla, V. Lulla, unpublished data). Therefore in our split helper system the helper-E construct used did not contain any capsid enhancer and still allowed efficient production of VRPs.

Alphaviruses are known to be transmitted by insect vectors, which exhibit body temperatures below $37^{\circ} \mathrm{C}$. Hence, the VRP production was also tested at lower temperature and has proven to be more efficient at $32^{\circ} \mathrm{C}$ than at $37^{\circ} \mathrm{C}$. Reducing the temperature to $28^{\circ} \mathrm{C}$ even slightly increased the VRP yield further but simultaneously slowed down the production process resulting in a prolonged production time (data not shown).

To facilitate and accelerate the readout of the NT assay we established a VRP system expressing Gluc, which is secreted into the supernatant [28]. Hence, measuring can directly be performed from the supernatant without the need of lysing cells. The latter was described to be necessary for the pseudotyped lentiviral vector-based CHIKV NT assay, which used Fluc as reporter protein [27]. In addition, the humanized Gluc has been described to be 1000-fold more sensitive compared to humanized Rluc or Fluc $[28,34]$. Furthermore, Gluc is very stable (half-life around 6 days [35]), which allows storage of the supernatant at $4{ }^{\circ} \mathrm{C}$ for several days without significantly loosing activity [28]. On the other hand, due to the high sensitivity, Gluc reporter assays are likely to be prone to pipetting errors [28]. Hence, pipetting smaller volumes when working in the 96-well format at lower MOI might be one reason for the higher deviations observed in this experimental setup. As already described, it is not recommended to use a pipetting volume below $10 \mu \mathrm{l}$ for Gluc assays [28]. Nevertheless, when using an MOI of 5, reliable results were also obtained in the 96-well format. Using an even higher MOI is not recommended. Besides consuming excessive amounts of VRPs, the risk to exhaust the assay exists. Also, especially at $24 \mathrm{~h}$ post infection, the amount of Gluc released into the supernatant after using an MOI of 50 was so high that samples had to be diluted to allow 
measurement of Gluc activity (data not shown). This could result in an additional source of variation.

The 96-well format might especially be favorable to use when only small sample volumes are available or when neutralizing titers have to be determined for an extensive number of samples. Working in a 96-well format allows to directly transfer the Gluc containing supernatant from the NT assay plate to a corresponding 96-well readout plate using a multichannel pipette thereby avoiding time consuming single pipetting steps. Furthermore, both the recently described pseudotyped lentiviral vector-based NT assay [27] and our VRP based NT assay have the advantage that the use of infectious CHIKV particles is avoided. However, for the lentiviral system readout is performed several days after transduction, whereas our VRP based assay allows carrying out the NT assay including readout within one day.

Performing different types of NT assays results in fairly different scales of NT titers. This was also observed when comparing the lentiviral vector-based NT assay with a classical plaque neutralization assay [27]. Similarly, the antibody dilutions for which the infectivity was inhibited by $50 \%$ were different for each sample comparing our VRP based assay with a plaque neutralization assay. Nevertheless, the order of neutralization potency among the samples was consistent (Table 1) indicating that the assay is suitable to determine comparative neutralization activities. Furthermore, the fact that no infectious CHIKV particles are needed and that readout can already be performed after $6 \mathrm{~h}$ makes the established Gluc VRP based NT assay a valuable tool to study patient or animal serum samples. Besides diagnostic purposes, analyses of neutralizing antibodies in human sera will help to understand immune mechanisms involved in CHIKV disease and analyses in animals will be useful in evaluation steps during CHIKV vaccine development.

\section{Conclusions}

We have established an NT assay based on CHIKV VRPs using secreted Gluc as readout. This circumvents the use of infectious CHIKV and allows an easy readout. The VRP based assay can be performed in microtiter plates and readout can be done within a single day making it suitable for high-throughput analyses of CHIKV neutralization antibodies in human or animal sera.

\section{Methods}

\section{Cells and viruses}

Baby hamster kidney-21 (BHK-21) cells were maintained in Glasgow's Minimum Essential Medium (GMEM) supplemented with $5 \%$ fetal bovine serum (FBS), 1\% Lglutamine, $10 \%$ tryptose phosphate broth, $20 \mathrm{mM}$ HEPES $\mathrm{pH} 7.2,100 \mathrm{U} / \mathrm{ml}$ penicillin and $0.1 \mathrm{mg} / \mathrm{ml}$ streptomycin at $37^{\circ} \mathrm{C}$ and $5 \% \mathrm{CO}_{2}$.
A stock of recombinant wild-type CHIKV (CHIKVLR2006 OPY1, [36]) was produced on BHK-21 cells. Cells were infected at an MOI of 0.1 for $1 \mathrm{~h}$ and virus harvested from the supernatant 2 days p.i. was stored at $-80^{\circ} \mathrm{C}$. Experiments with infectious CHIKV were performed in a biosafety level 3 laboratory.

\section{Antibodies and antisera}

Monoclonal antibody D3.62 $(35 \mathrm{mg} / \mathrm{ml})$ is directed against CHIKV E2 protein (T. Couderc and M. Lecuit, unpublished data). Human sera containing neutralizing CHIKV antibodies were obtained from patients returning to Europe from the Indian Ocean region in 2006 and have been described previously [30]. Briefly, sera from patient 575/06 returned from the Seychelles, patient 662/06 from La Réunion and patient 1753/06 from Mauritius. Monoclonal antibody Z2G2 recognizing CHIKV capsid protein was kindly provided by Petra Emmerich (Bernhard Nocht Institute, Hamburg, Germany).

\section{Indirect immunofluorescence}

BHK-21 cells were cultured on glass coverslips and infected with CHIKV-LR2006 OPY1 at an MOI of 0.5. At $24 \mathrm{~h}$ after infection, cells were fixed with ice-cold methanol / acetone (1:1) and air-dried. Serum samples or monoclonal antibodies were diluted 1:5000 in PBS and incubated for $1 \mathrm{~h}$ at $37^{\circ} \mathrm{C}$ on fixed cells. Serum antibodies were detected by Alexa 488-labeled goat anti-human IgG (Jackson ImmunoResearch, 1:500) and monoclonal antibodies by cyanine 3 -conjugated goat anti-mouse IgG (Jackson ImmunoResearch, 1:200) using fluorescence microscopy (Axiovert 40 microscope, Zeiss). Nuclei were stained with 4,6-diamidino-2-phenylindole (DAPI).

\section{Plasmid constructs}

The construction of full-length infectious cDNA of CHIKV-LR2006 OPY1 is described elsewhere [36]. To obtain plasmid pChikRepl containing the cDNA of a CHIKV replicon, the region of the infectious cDNA clone corresponding to the coding sequence of CHIKV structural proteins (nucleotides 7565-11310 of CHIKV-LR2006 OPY1) was replaced with sequence 5' CCTAGGTAAT AAGTTTAAAC 3' (recognition sites of restriction endonucleases AvrII and MssI (PmeI) are underlined) by PCRmediated mutagenesis. The coding sequence of Gluc (optimized for human codon usage, synthesized by GeneArt (Life Technologies)) was PCR amplified using primers 5' TATTCCTAGGCCACCATGGGAGTCAAAG TTCTGTTTGCC 3' (start codon is in bold, recognition site of AvrII restriction enzyme is underlined) and 5' TGATGTTTAAACTTAGTCACCACCGGCCCCCTTG ATCTT 3' (stop codon is in bold, recognition site of MssI restriction enzyme is underlined); the obtained fragment was digested with AvrII and MssI enzymes 
(Thermo Scientific, USA) and inserted into pChikRepl vector digested with the same enzymes. The resulting plasmid was designated as pChikRepl-Gluc. To obtain a plasmid, containing the cDNA of a packaging construct for ChikRepl-Gluc, the region of the infectious cDNA corresponding to nucleotides 308-7419 of CHIKVLR2006 OPY1 [36] was replaced by sequence 5' GTTTAAAC 3' (recognition site of MssI restriction enzyme) using PCR-mediated mutagenesis; the resulting plasmid was designated pChikHelper. To obtain plasmids for a split helper system the following changes were introduced into pChikHelper using PCR based mutagenesis. First, to obtain pChikHelper-E the region corresponding to nucleotides 461-1248 (coding region for capsid protein) of pChikHelper was replaced by sequence 5' CCTAGGCCACCATG 3' (recognition site of AvrII restriction enzyme is underlined). Second, to obtain pChikHelper-C the region corresponding to nucleotides 1246-4206 (coding region for E3-E2-E1 glycoproteins) of pChikHelper was replaced by sequence $5^{\prime}$ TAAGTTTAAAC 3' (recognition site of MssI restriction enzyme is underlined). All constructs were verified using Sanger sequencing. Sequences of all plasmid vectors are available from authors upon request.

\section{Electroporation and VRP production}

Replicon and helper DNA templates were linearized with NotI and in vitro transcribed using the mMESSAGE mMACHINE SP6 Kit (Ambion). For recovery of VRPs 1 $\mu \mathrm{g}$ of each, the replicon and helper RNAs, were coelectroporated into $1 \times 10^{6}$ BHK-21 cells using BHK21 preset protocol of Gene Pulser Xcell (Bio-Rad). Cells were seeded into $25 \mathrm{~cm}^{2}$ flasks and incubated at $32^{\circ} \mathrm{C}$ for $36 \mathrm{~h}$. For large-scale VRP production supernatants of six electroporations were pooled for purification. After removing detached cells and cell debris by clarifying for $30 \mathrm{~min}$ at $4000 \mathrm{~g}$ and $4{ }^{\circ} \mathrm{C}$, the supernatants were passed through a $0.45 \mu \mathrm{m}$ filter and applied to a $20 \%$ sucrose cushion followed by centrifugation for $90 \mathrm{~min}$ at 25000 rpm and $4^{\circ} \mathrm{C}$ (SW 32 Ti rotor, Beckman Coulter). Pellets were resuspended in TNE buffer $(50 \mathrm{mM}$ Tris- $\mathrm{HCl}, \mathrm{pH}$ 7.4, $100 \mathrm{mM} \mathrm{NaCl}, 0.5 \mathrm{mM}$ EDTA) over night at $4^{\circ} \mathrm{C}$ before passing through a $0.22 \mu \mathrm{m}$ filter. VRPs were stored at $-80^{\circ} \mathrm{C}$.

\section{Real-time reverse transcription-PCR}

RNA from VRPs in the cell culture supernatant or after sucrose cushion purification was extracted using NucleoSpin RNA Virus Kit (Macherey-Nagel). The isolated RNA was detected by real-time reverse transcription-PCR (RT-PCR) using the SuperScript III One-Step RT-PCR System with Platinum Taq DNA polymerase (Invitrogen). For detection of CHIKV RNA, the $25 \mu \mathrm{l}$ reaction contained $3 \mu \mathrm{l}$ of RNA, 1x Reaction Mix, $0.5 \mu \mathrm{g}$ BSA, $0.5 \mu \mathrm{l}$ SS III RT / Platinum
Taq Mix, $0.6 \mu \mathrm{M}$ of primer CHIKSI, $0.6 \mu \mathrm{M}$ of primer CHIKASI and $0.2 \mu \mathrm{M}$ of the CHIKP probe [30]. Thermocycling was performed on a LightCycler 480 (Roche) programmed for: $30 \mathrm{~min}$ at $50^{\circ} \mathrm{C}$ for reverse transcription, 2 min at $94^{\circ} \mathrm{C}$ to activate the $\mathrm{Taq}$ polymerase and $45 \mathrm{PCR}$ amplification cycles of $15 \mathrm{sec}$ at $94^{\circ} \mathrm{C}, 30 \mathrm{sec}$ at $58^{\circ} \mathrm{C}$ and $30 \mathrm{sec}$ at $72^{\circ} \mathrm{C}$. Photometrically quantified in vitro-RNA transcripts of the target regions were used in the PCR to generate a standard curve for viral RNA quantification.

\section{Luciferase assay}

Gluc was measured from the supernatant of infected cells using Renilla Luciferase Assay System (Promega) according to the manufacturer's instructions. Luciferase activity was measured in RLUs. Measurement was performed automated in a Synergy 2 microplate reader (BioTek) using polystyrol microplates (Greiner Bio-one).

\section{VRP neutralization assay}

The day before infection, 24- or 96-well plates were seeded with $1 \times 10^{5}$ or $2 \times 10^{4}$ BHK-21 cells per well, respectively. VRPs were applied in the NT assay at MOI 5 , 0.5 or 0.05 (calculated based on VRP RNA copies $/ \mathrm{ml}$ ). All dilutions were performed using GMEM supplemented with $1 \%$ FBS. VRP dilutions were incubated with serially diluted antibody or human serum for $1 \mathrm{~h}$ at $37^{\circ} \mathrm{C}$ before adding the mixture to a monolayer of BHK-21 cells in either a 24-well plate (total volume per well $200 \mu \mathrm{l}$ ) or a 96-well plate (total volume per well $40 \mu \mathrm{l}$ ). After incubation for $1 \mathrm{~h}$ at $37^{\circ} \mathrm{C}$ the inoculum was removed, cells were washed once with PBS and medium was added. Supernatants for Gluc measurement were taken at $6 \mathrm{~h}$ and $24 \mathrm{~h} \mathrm{p}$. i. Neutralization potency was determined as percentage of measured Gluc activity compared to Gluc readout after VRP application without antibody/serum.

\section{Plaque reduction neutralization assay}

About 100 PFU of infectious wild-type virus were incubated with serially diluted antibody or human serum for $1 \mathrm{~h}$ at $37^{\circ} \mathrm{C}$, added to a monolayer of BHK-21 cells in a 6-well plate (total volume per well $600 \mu \mathrm{l}$ ) and incubated at $37^{\circ} \mathrm{C}$ for $1 \mathrm{~h}$. Subsequently, the inoculum was replaced by an overlay containing $0.6 \%$ agarose in MEM. At $48 \mathrm{~h}$ p.i. cells were fixed with $7 \%$ formaldehyde and plaques were visualized using crystal violet staining (1\% crystal violet in $50 \%$ ethanol). Neutralization potency was determined as percentage of plaque titers compared to plaque titers after virus infection without antibody/serum.

\section{Statistics}

Probit analysis for determination of $\mathrm{NT}_{50}$ values was done with the SPSSV21 software package (IBM, Ehningen, Germany). 


\section{Competing interests}

The authors declare that they have no competing interests.

\section{Authors' contributions}

SG, BMK conceived and designed experiments; AL, VL, AM designed and established constructs for VRP production; TC, ML, CD contributed CHIKV antibody/sera; JFD performed statistical analyses; PL participated in optimized VRP production; SG, AM, BMK wrote the paper. All authors have read and approved the manuscript.

\section{Acknowledgments}

We thank Janett Wieseler for excellent technical assistance and Ionna Dimitriou for help with statistical analyses. This work was supported by the European Union FP7 project "Integrated Chikungunya Research" (ICRES; grant no. 261202), Institut Pasteur, Inserm, Ville de Paris, Fondation BNP-Paribas, and LabEx IBEID.

\section{Author details \\ ${ }^{1}$ Institute of Virology, University of Bonn Medical Centre, Bonn, Germany. ${ }^{2}$ Institute of Technology, University of Tartu, Tartu, Estonia. ${ }^{3}$ Institut Pasteur, Biology of Infection Unit, Paris, France. ${ }^{4}$ Inserm U1117, Paris, France. ${ }^{5}$ Department of Microbiology, Tumor and Cell Biology, Karolinska Institutet, Stockholm, Sweden. 'Sorbonne Paris Cité, Institut Imagine, Paris Descartes University, Paris, France.}

Received: 10 March 2013 Accepted: 3 July 2013

Published: 15 July 2013

\section{References}

1. Kuhn R: Togaviridae: the viruses and their replicatioon. In Fields Virology, Volume 1. 5th edition. Edited by Knipe DM, Howley PM. Philadelphia: Lippincott Williams \& Wilkons; 2007:1001-1022.

2. Frolov I, Hoffman TA, Pragai BM, Dryga SA, Huang HV, Schlesinger S, Rice CM: Alphavirus-based expression vectors: strategies and applications. Proc Natl Acad Sci USA 1996, 93:11371-11377.

3. Liljeström P, Garoff H: A new generation of animal cell expression vectors based on the Semliki Forest virus replicon. Biotechnology (N Y) 1991, 9:1356-1361.

4. Pushko P, Parker M, Ludwig GV, Davis NL, Johnston RE, Smith JF: Repliconhelper systems from attenuated Venezuelan equine encephalitis virus: expression of heterologous genes in vitro and immunization against heterologous pathogens in vivo. Virology 1997, 239:389-401.

5. Xiong C, Levis R, Shen P, Schlesinger S, Rice CM, Huang HV: Sindbis virus: an efficient, broad host range vector for gene expression in animal cells. Science 1989, 243:1188-1191.

6. Smerdou C, Liljeström P: Two-helper RNA system for production of recombinant Semliki forest virus particles. J Virol 1999, 73:1092-1098.

7. Frolov I, Frolova E, Schlesinger S: Sindbis virus replicons and Sindbis virus: assembly of chimeras and of particles deficient in virus RNA. J Virol 1997, 71:2819-2829.

8. Robinson MC: An epidemic of virus disease in Southern Province, Tanganyika Territory, in 1952-53. I. Clinical features. Trans R Soc Trop Med Hyg 1955, 49:28-32.

9. Ross RW: The Newala epidemic. III. The virus: isolation, pathogenic properties and relationship to the epidemic. J Hyg (Lond) 1956, 54:177-191.

10. Enserink M: Infectious diseases. Massive outbreak draws fresh attention to little-known virus. Science 2006, 311:1085.

11. Charrel RN, de Lamballerie X, Raoult D: Chikungunya outbreaks-the globalization of vectorborne diseases. N Engl J Med 2007, 356:769-771.

12. D'Ortenzio E, Grandadam M, Balleydier E, Dehecq JS, Jaffar-Bandjee MC, Michault A, Andriamandimby SF, Reynes JM, Filleul L: Sporadic cases of chikungunya, Reunion Island, August 2009. Euro Surveill 2009, 14(35).

13. Paupy C, Kassa Kassa F, Caron M, Nkoghé D, Leroy EM: A chikungunya outbreak associated with the vector Aedes albopictus in remote villages of Gabon. Vector Borne Zoonotic Dis 2012, 12:167-169.

14. Renault P, Balleydier E, D'Ortenzio E, Baville M, Filleul L: Epidemiology of Chikungunya infection on Reunion Island, Mayotte, and neighboring countries. Med Mal Infect 2012, 42:93-101.

15. Rezza G, Nicoletti L, Angelini R, Romi R, Finarelli AC, Panning M, Cordioli P, Fortuna C, Boros S, Magurano F, Silvi G, Angelini P, Dottori M, Ciufolini MG, Majori GC, Cassone A, CHIKV study group: Infection with chikungunya virus in Italy: an outbreak in a temperate region. Lancet 2007, 370:1840-1846.

16. Grandadam M, Caro V, Plumet S, Thiberge JM, Souares Y, Failloux AB, Tolou HJ, Budelot M, Cosserat D, Leparc-Goffart I, Despres P: Chikungunya virus, southeastern France. Emerg Infect Dis 2011, 17:910-913.

17. Diallo M, Thonnon J, Traore-Lamizana M, Fontenille D: Vectors of Chikungunya virus in Senegal: current data and transmission cycles. Am J Trop Med Hyg 1999, 60:281-286.

18. Kumar NP, Sabesan S, Krishnamoorthy K, Jambulingam P: Detection of Chikungunya virus in wild populations of Aedes albopictus in Kerala State, India. Vector Borne Zoonotic Dis 2012, 12:907-911.

19. Tsetsarkin KA, Vanlandingham DL, McGee CE, Higgs S: A single mutation in chikungunya virus affects vector specificity and epidemic potential. PLoS Pathog 2007, 3:e201.

20. Borgherini G, Poubeau P, Staikowsky F, Lory M, Le Moullec N, Becquart JP, Wengling C, Michault A, Paganin F: Outbreak of chikungunya on Reunion Island: early clinical and laboratory features in 157 adult patients. Clin Infect Dis 2007, 44:1401-1407.

21. Brighton SW, Prozesky OW, de la Harpe AL: Chikungunya virus infection. A retrospective study of 107 cases. S Afr Med J 1983, 63:313-315.

22. Kam YW, Simarmata D, Chow A, Her Z, Teng TS, Ong EK, Renia L, Leo YS, $\mathrm{Ng}$ LF: Early appearance of neutralizing immunoglobulin G3 antibodies is associated with chikungunya virus clearance and long-term clinical protection. J Infect Dis 2012, 205:1147-1154

23. Kumar M, Sudeep AB, Arankalle VA: Evaluation of recombinant E2 proteinbased and whole-virus inactivated candidate vaccines against chikungunya virus. Vaccine 2012, 30:6142-6149.

24. Kam YW, Lum FM, Teo TH, Lee WW, Simarmata D, Harjanto S, Chua CL, Chan YF, Wee JK, Chow A, Lin RT, Leo YS, Le Grand R, Sam IC, Tong JC, Roques P, Wiesmüller KH, Rénia L, Rötzschke O, Ng LF: Early neutralizing lgG response to Chikungunya virus in infected patients targets a dominant linear epitope on the E2 glycoprotein. EMBO Mol Med 2012, 4:330-343.

25. Khan M, Dhanwani R, Rao PV, Parida M: Subunit vaccine formulations based on recombinant envelope proteins of Chikungunya virus elicit balanced Th1/Th2 response and virus-neutralizing antibodies in mice. Virus Res 2012, 167:236-246.

26. Wang E, Volkova E, Adams AP, Forrester N, Xiao SY, Frolov I, Weaver SC: Chimeric alphavirus vaccine candidates for chikungunya. Vaccine 2008, 26:5030-5039.

27. Kishishita N, Takeda N, Anuegoonpipat A, Anantapreecha S: Development of a Pseudotyped Lentiviral Vector-Based Neutralization Assay for Chikungunya Virus Infection. J Clin Microbiol 2013, 51:1389-1395.

28. Tannous BA: Gaussia luciferase reporter assay for monitoring biological processes in culture and in vivo. Nat Protoc 2009, 4:582-591.

29. Verhaegent M, Christopoulos TK: Recombinant Gaussia luciferase. Overexpression, purification, and analytical application of a bioluminescent reporter for DNA hybridization. Anal Chem 2002, 74:4378-4385.

30. Panning M, Grywna K, van Esbroeck M, Emmerich P, Drosten C: Chikungunya fever in travelers returning to Europe from the Indian Ocean region, 2006. Emerg Infect Dis 2008, 14:416-422.

31. Berglund P, Sjoberg M, Garoff H, Atkins GJ, Sheahan BJ, Liljestrom P: Semliki Forest virus expression system: production of conditionally infectious recombinant particles. Biotechnology (N Y) 1993, 11:916-920.

32. Frolov I, Schlesinger S: Translation of Sindbis virus mRNA: effects of sequences downstream of the initiating codon. J Virol 1994, 68:8111-8117.

33. Sjöberg EM, Suomalainen M, Garoff H: A significantly improved Semliki Forest virus expression system based on translation enhancer segments from the viral capsid gene. Biotechnology (N Y) 1994, 12:1127-1131.

34. Tannous BA, Kim DE, Fernandez JL, Weissleder R, Breakefield XO: Codonoptimized Gaussia luciferase cDNA for mammalian gene expression in culture and in vivo. Mol Ther 2005, 11:435-443.

35. Wurdinger T, Badr C, Pike L, de Kleine R, Weissleder R, Breakefield XO, Tannous BA: A secreted luciferase for ex vivo monitoring of in vivo processes. Nat Methods 2008, 5:171-173.

36. Pohjala L, Utt A, Varjak M, Lulla A, Merits A, Ahola T, Tammela P: Inhibitors of alphavirus entry and replication identified with a stable Chikungunya replicon cell line and virus-based assays. PLoS One 2011, 6:e28923.

\section{doi:10.1186/1743-422X-10-235}

Cite this article as: Gläsker et al:: Virus replicon particle based Chikungunya virus neutralization assay using Gaussia luciferase as readout. Virology Journal 2013 10:235. 